\title{
Removal of Low Density Lipoprotein from Blood Plasma using Cross-linked, Sulfated Polyvinylalcohol
}

\author{
N. Maaskant ${ }^{1}$, A. Bantjes ${ }^{1}$ and H.J.M. Kempen ${ }^{2}$ \\ 'Twente University of Technology, Department of Chemical Technolog), P.O. Box 217, 7500 AE Enschede, and \\ 'TNO Gaubius Institute, Herenstraat 5d, 2313 AD Leiden (The Netherlands) \\ (Received 25 June, 1985) \\ (Revised, received 27 February and 10 May 1986) \\ (Accepted 23 June, 1986)
}

\begin{abstract}
Summary
The properties of a new synthetic LDL binding material, consisting of fragments of loosely crosslinked hydrogel, based on sulfated polyvinylalcohol are described. When incubated with 20 times its volume of plasma, this material binds up to $95 \%$ of the LDL, even from plasma with severely elevated LDL cholesterol levels (up to $20 \mathrm{mM}$ ). In addition a cholesterol-rich subfraction of VLDL is bound but HDL is not bound. After about $10 \mathrm{~min}$ binder/plasma contact the LDL removal is complete and no other additives are required. LDL binding capacity is dependent on the average binder particle size, indicating a restricted penetration of LDL particles into the binder matrix.
\end{abstract}

Key words: Familial hypercholesterolemia - LDL selective removal - Sulfated hydrogel

\section{Introduction}

Familial hypercholesterolemia (FH) is a genetically inherited metabolic disorder, characterized by a severely increased level of plasma low density lipoproteins (LDL) (heterozygotes usually about

This work was supported financially by Organon Teknika NV. Turnhout, Belgium.

Correspondence and reprint requests to: Prof $\mathrm{dr}$. A. Bantjes, University of Technology, Department of Chemical Technology, P.O. Box 217, 7500 AE Enschede. The Netherlands.

Abbreviations: $\mathrm{FH}=$ familial hypercholesterolemia. $\mathrm{HLP}=$ hyperlipoproteinemia, $\mathrm{LDL}=$ low density lipoprotein, $\mathrm{PBS}=$ phosphate-buffered saline, and PVAS = sulfated polyvinylalcohol
2 -fold normal, homozygotes up to 10 -fold normal). These increased plasma LDL levels are caused by a lack of specific cell surface LDL receptors. Elevated LDL levels present an increased risk of the occurrence of atherosclerosis. Homozygote patients often die from a myocardial infarction before age 20 . Atherosclerotic disease affects $40 \%$ of heterozygote males and $20 \%$ of heterozygote females before the age of 40 , being fatal for almost a quarter of the males before they reach age 50 [1].

A number of dietary and medical treatments reduce plasma LDL levels in $\mathrm{FH}$ patients to acceptable levels, especially when given in combination [2]. However, some medicines are not effective in homozygotes and most have unpleasant 
side-effects. An alternative method, used in patients with persistent hypercholesterolemia, consists of extracorporeal blood treatment, performed either as plasma exchange [3] or by means of more selective techniques for LDL removal [4-6]. The latter has been accomplished by perfusion of plasma or even full blood over columns or batches of immobilized heparin [4] or LDL antibodies [5], or by precipitation of LDL using buffered heparin [6].

In the present paper we report on the preparation and properties of a new LDL binding material, based on sulfated polyvinylalcohol. This material may offer a suitable and more economic alternative for the above-mentioned sepharose-based LDL sorbents.

\section{Materials and Methods}

Polyvinylalcohol (PVA) (MW 14000-86000, obtained from Janssen Chimica, Beerse, Belgium) was sulfated by reaction with a pyridine- $\mathrm{SO}_{3}$ complex in excess pyridine (obtained from Merck, Darmstadt, F.R.G.) (molar ratio pyridine$\mathrm{SO}_{3} / \mathrm{PVA}=1.3 / 1$ ), for 90 min at $105^{\circ} \mathrm{C}$ (molar ratio pyridine $/ \mathrm{PVA}=1 / 1$ ). The pyridine- $\mathrm{SO}_{3}$ complex was prepared by adding chlorosulfonic acid (obtained from Merck, Darmstadt, F.R.G.) dropwise to cooled pyridine (1 mole chlorosulfonic acid $/ 2$ moles pyridine). After the sulfation reaction the reaction mixture was diluted in distilled water and neutralized with $\mathrm{NaOH}$; the pyridine was distilled off under vacuum and the sulfated PVA (PVAS) was purified by dialysis against distilled water.

A dry product was obtained either by ethanol precipitation or by freeze-drying the dialyzed PVAS solution. A loosely cross-linked hydrogel was obtained by $\gamma$-irradiation of aqueous solutions of PVAS in glass tubes of $8 \mathrm{~mm}$ diameter. Irradiation dose was varied between 25 and 100 kGy $\left({ }^{60} \mathrm{Co}\right.$, dose rate $\left.3.5 \mathrm{kGy} / \mathrm{h}\right)$, PVAS concentrations between 10 and $60 \%(w / w)$.

After irradiation the hydrogels were pushed from the tubes and thoroughly washed with distilled water. After this washing step (accompanied by extensive swelling; see Results) the PVAS hydrogel was fragmented by one of the two following procedures: (1) freeze-drying of the gel fol- lowed by milling and sieving of the (dry) gel particles. By this method gel particles with a narrow particle size distribution were obtained (sieve fractions: $<45 \mu \mathrm{m} ; 45-53 \mu \mathrm{m} ; 53-63 \mu \mathrm{m} ; 63-90$ $\mu \mathrm{m} ; 90-105 \mu \mathrm{m})$. (2) Equilibration of the hydrogel in PBS, followed by fragmentation in a Dounce homogenizer, yielded particle sizes between 50 and $250 \mu \mathrm{m}$ after reequilibration in PBS. Unless otherwise indicated the latter fragmentation method was used.

Varying amounts of serum or of citrated or heparinized human plasma from healthy volunteers or from different hyperlipoproteinemic patients were incubated for $30 \mathrm{~min}$ with a constant volume of PVAS hydrogel particles, at $37^{\circ} \mathrm{C}$ in polystyrene tubes, which were mounted in a slowly rotating apparatus. Thereafter, the tubes were centrifuged for $10 \mathrm{~min}$ at $1000 \times \mathrm{g}$ to sediment the gel particles.

The lipoprotein binding was studied either by: (1) determination of the total plasma cholesterol concentration before and after incubation or (2) isolation of the different lipoproteins (VLDL + IDL, LDL, HDL) by density gradient ultra centrifugation [7] before and after incubation of the whole plasma with the gel fragments, followed hy determination of the lipoprotein concentrations by measuring the amount of cholesterol and triglyceride in each lipoprotein fraction. The fractions comprised VLDL + IDL $(d=1.006-1.019$ $\mathrm{g} / \mathrm{ml}), \mathrm{LDL}(d=1.019-1.060 \mathrm{~g} / \mathrm{ml}), \mathrm{HDL}_{2}(d=$ $1.060-1.130 \mathrm{~g} / \mathrm{ml})$ and $\mathrm{HDL}_{3}(d=1.130-1.210$ $\mathrm{g} / \mathrm{ml})$.

Cholesterol concentrations were determined according to [8]. Triglyceride concentrations were determined according to [9]. Binding was calculated from the difference in concentration in the fluid phase before and after incubation with the absorbent. $\mathrm{Mg}^{2+}$ and $\mathrm{Ca}^{2+}$ concentrations were determined by standard atomic absorption techniques. $L p(a)$ and antithrombin-III (AT-III) concentrations were determined by electro immunoassay $[10]$ and apo $B$ by radial immuno diffusion [11]. Albumin and $\alpha_{1}-, \alpha_{2}, \beta$ - and $\gamma$-globulins concentrations were determined by electrophoresis. Fibrinogen (Fb) and fibrin(ogen) degradation products (FDP/fdp) were quantitated by means of an enzyme-immunoassay, newly developed in collaboration with Organon Teknika. For Fb, 
monoclonal antibody $\mathrm{Y}-18$ was used as tagging [12] antibody and a non-specific monoclonal antibody, recognizing fibrinogen in the D-domain, as catching antibody. For FDP/fdp, the monoclonal antibody FDP-14 [13] served as catching, and the non-specific anti-D monoclonal as tagging antibody. All incubations were performed in duplicate, and were repeated unless the duplicate values differed less than $10 \%$.

\section{Results}

\section{Sulfation}

Depending on the molar ratio pyridine- $\mathrm{SO}_{3}$ complex/PVA hydroxyl groups the substitution of the PVA hydroxyl groups by sulfate groups is 60-95\%. The experiments described were carried out with $80 \%$ sulfated PVA.

\section{Hydrogel formation}

After $\gamma$-irradiation the binder contains $40-85 \%$ water. During the washing and equilibration in PBS the binder swells, due to osmotic pressure effects, to a water content of $95-99 \%$.

Table 1 shows the influence of both irradiation dose and PVAS concentration during radiation on the water content after equilibration in PBS. At higher water contents the hydrogels are more loosely cross-linked, improving the penetration of LDL particles into the binder matrix. A relatively low water content on the other hand indicates a high cross-linking density and therefore the possibility of a too finely meshed network inhibiting LDL penetration.

\section{Lipoprotein binding to PVAS-hydrogelparticles}

Upon incubation of PVAS-particles with 20

\section{TABLE 1}

PERCENTAGE CONTENT OF WATER AT SWELLING EQUILIBRIUM IN PBS OF PVAS HYDROGELS (DEGREE OF SULFATION PVAS $=0.73$ )

\begin{tabular}{llll}
\hline \multirow{2}{*}{$\begin{array}{l}\text { Irradiation dose } \\
\text { (kGy) }\end{array}$} & \multicolumn{3}{c}{ \% PVAS during } \\
\cline { 2 - 4 }$\gamma$-irradiation $(w / w)$ \\
\hline 28 & 10 & 15 & 20 \\
\hline 56 & 98.7 & 97.9 & 95.4 \\
90 & 98.3 & 97.5 & 95.5 \\
118 & 98.3 & 96.2 & 95.2 \\
\hline
\end{tabular}

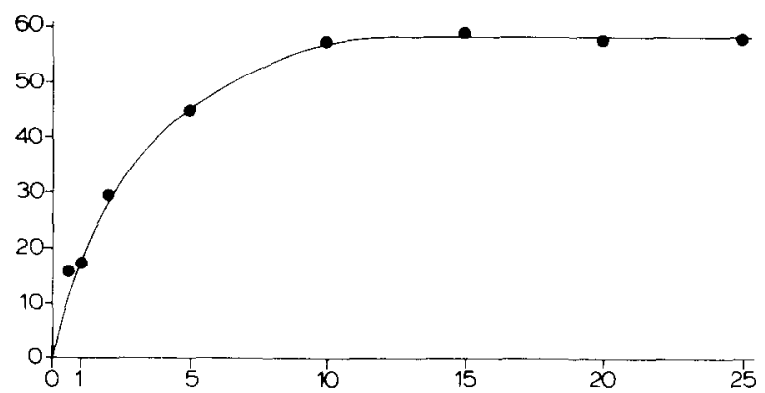

Fig. 1. Influence of incubation time on cholesterol binding from pooled citrated plasma by fragments of cross linked PVAS ( $5 \%$ by volume). Cholesterol concentration in this plasma: $4.3 \mathrm{mM}$; LDL cholesterol: $2.3 \mathrm{mM}$.

times its volume of plasma (from a pool composed of plasma of 5 male, normolipidemic volunteers aged 25-37, not suffering from any metabolic endocrine or infectious disease), the particles removed about $60 \%$ of the total amount of cholesterol within $10 \mathrm{~min}$ (Fig. 1). This is equivalent to the total amount of cholesterol in the LDL fraction in that plasma. Table 2 shows lipoprotein profiles before and after incubation of 1 volume binder particles with 20 volumes of citrated normal or hyperlipoproteinemic plasma.

From these results it can be concluded that: (1) LDL is bound by 56-95\%; (2) in case of HLP type IIa or b virtually all the LDL is bound; (3) VLDL is not, or for a much smaller percentage, bound in comparison to LDL. However, the percent cholesterol bound is always higher than the percent triglycerides bound from this fraction, indicating preferential binding of a cholesterol-rich subfraction of VLDL; (4) an extremely elevated VLDL concentration might reduce the LDL binding capacity (type IV plasma); (5) $\mathrm{HDL}_{2}$ and $\mathrm{HDL}_{3}$ are not bound. In addition, the gel was found to bind $L p(a)$ antigen from whole serum containing a high level of this antigen with a similar efficiency as apo B (Table 3 ).

Figure 2 shows the results of an experiment in which the LDL binder was incubated in varying binder/plasma ratios with 3 different plasmas with different total cholesterol levels and lipoprotein spectra. The LDL binder has a very high capacity for LDL binding; even when the binder is incubated with 10-20 times its own volume of plasma containing an extremely elevated choles- 
terol level $(20.7 \mathrm{mM}) 70-80 \%$ of the total amount of cholesterol is removed, equivalent to $80-95 \%$ of LDL cholesterol (Fig. 2A). The absolute amount of bound cholesterol increases with increasing total plasma cholcsterol concentration and with increasing amounts of plasma a maximum is reached at a

TABLE 2

LIPOPROTEIN PROFILES, BEFORE AND AFTER INCUBATION OF PVAS HYDROGEL PARTICLES WITH VARIOUS CITRATED PLASMAS, OBTAINED FROM FASTING SUBJECTS

Plasma/binder ratio $20 / 1(\mathrm{v} / \mathrm{v})$.

\begin{tabular}{|c|c|c|c|c|c|c|c|}
\hline \multirow{2}{*}{$\begin{array}{l}\text { FH } \\
\text { fenotype }\end{array}$} & & \multicolumn{3}{|c|}{ Cholesterol } & \multicolumn{3}{|c|}{ Triglycerides } \\
\hline & & $\begin{array}{l}\text { Before } \\
(\mathrm{mmol} / \mathrm{l})\end{array}$ & $\begin{array}{l}\text { After } \\
(\mathrm{mmol} / 1)\end{array}$ & $\begin{array}{l}\% \text { bound } \\
(\mathrm{mmol} / \mathrm{l})\end{array}$ & $\begin{array}{l}\text { Before } \\
(\mathrm{mmol} / \mathrm{l})\end{array}$ & $\begin{array}{l}\text { After } \\
(\mathrm{mmol} / 1)\end{array}$ & $\begin{array}{l}\text { \% bound } \\
(\mathrm{mmol} / \mathrm{l})\end{array}$ \\
\hline \multirow[t]{2}{*}{ Normal } & VLDL & 1.11 & 0.41 & 63 & 0.61 & 0.33 & 46 \\
\hline & LDL & 1.84 & 0.22 & 88 & 0.18 & 0.04 & 78 \\
\hline \multirow[t]{4}{*}{ (male, 37) } & $\mathrm{HDL}_{2}$ & 0.26 & 0.28 & NS & 0.05 & 0.06 & NS \\
\hline & $\mathrm{HDL}_{3}$ & 0.60 & 0.64 & NS & 0.08 & 0.09 & NS \\
\hline & Rest & 0.10 & 0.11 & NS & 0.01 & 0.01 & NS \\
\hline & Total & 3.91 & 1.66 & 58 & 0.93 & 0.53 & 43 \\
\hline \multirow[t]{2}{*}{ II a } & VLDL & 1.88 & 1.68 & 11 & 1.95 & 2.11 & NS \\
\hline & IDL & 1.79 & 0.34 & 81 & 0.65 & 0.10 & 85 \\
\hline \multirow[t]{5}{*}{ (male, 49) } & LDL & 3.47 & 0.97 & 72 & 0.59 & 0.20 & 66 \\
\hline & $\mathrm{IIDL}_{2}$ & 0.11 & 0.06 & NS & 0.06 & 0.04 & NS \\
\hline & $\mathrm{HDL}_{3}$ & 0.35 & 0.33 & NS & 0.14 & 0.13 & NS \\
\hline & Rest & 0.09 & 0.11 & NS & 0.04 & 0.05 & NS \\
\hline & Total & 7.69 & 3.49 & 55 & 3.43 & 2.62 & 23 \\
\hline \multirow[t]{3}{*}{ II a } & VLDL & 1.13 & 0.29 & 74 & 1.05 & 0.56 & 47 \\
\hline & LDL & 6.79 & 0.31 & 95 & 0.45 & 0.01 & 98 \\
\hline & $\mathrm{HDL}_{2}$ & 0.07 & 0.09 & NS & 0.01 & 0.01 & NS \\
\hline \multirow[t]{3}{*}{ (male, 34) } & $\mathrm{HDI}_{3}$ & 0.51 & 0.50 & NS & 0.02 & 0.05 & NS \\
\hline & Rest & 0.12 & 0.10 & NS & 0.02 & 0.01 & NS \\
\hline & Total & 8.62 & 1.29 & 85 & 1.55 & 0.64 & 59 \\
\hline \multirow[t]{2}{*}{$11 \mathrm{a}$} & VLDL & 1.41 & 0.30 & 79 & 0.37 & 0.22 & 41 \\
\hline & LDL & 10.40 & 0.98 & 91 & 0.60 & 0.03 & 95 \\
\hline \multirow[t]{4}{*}{ (female, 64) } & $\mathrm{HDL}_{2}$ & 0.50 & 0.47 & NS & 0.04 & 0.05 & NS \\
\hline & $\mathrm{HDL}_{3}$ & 0.76 & 0.80 & NS & 0.06 & 0.07 & NS \\
\hline & Rest & 0.11 & 0.11 & NS & 0.00 & 0.01 & NS \\
\hline & Total & 13.18 & 2.66 & 80 & 1.07 & 0.38 & 64 \\
\hline \multirow[t]{2}{*}{ IIb } & VLDL & 2.55 & 0.62 & 76 & 1.47 & 1.01 & 31 \\
\hline & LDL & 6.90 & 0.37 & 95 & 0.56 & 0.10 & 82 \\
\hline \multirow[t]{4}{*}{ (female, 42) } & $\mathrm{HDL}_{2}$ & 0.22 & 0.22 & NS & 0.05 & 0.05 & NS \\
\hline & $\mathrm{HDL}_{3}$ & 0.58 & 0.59 & NS & 0.14 & 0.17 & NS \\
\hline & Rest & 0.09 & 0.08 & NS & 0.03 & 0.01 & NS \\
\hline & Total & 10.34 & 1.88 & 82 & 2.25 & 1.34 & 40 \\
\hline \multirow[t]{2}{*}{ IV } & VLDL & 11.49 & 8.77 & 24 & 10.41 & 7.86 & 24 \\
\hline & LDL & 2.36 & 1.03 & 56 & 0.38 & 0.22 & 42 \\
\hline \multirow[t]{4}{*}{ (male, 46) } & $\mathrm{HDL}_{2}$ & 0.26 & 0.24 & NS & 0.06 & 0.07 & NS \\
\hline & $\mathrm{HDL}_{3}$ & 0.76 & 0.81 & NS & 0.13 & 0.15 & NS \\
\hline & Rest & 0.09 & 0.09 & NS & 0.01 & 0.01 & NS \\
\hline & Total & $\overline{14.96}$ & 10.94 & 27 & 10.99 & 8.31 & 24 \\
\hline
\end{tabular}



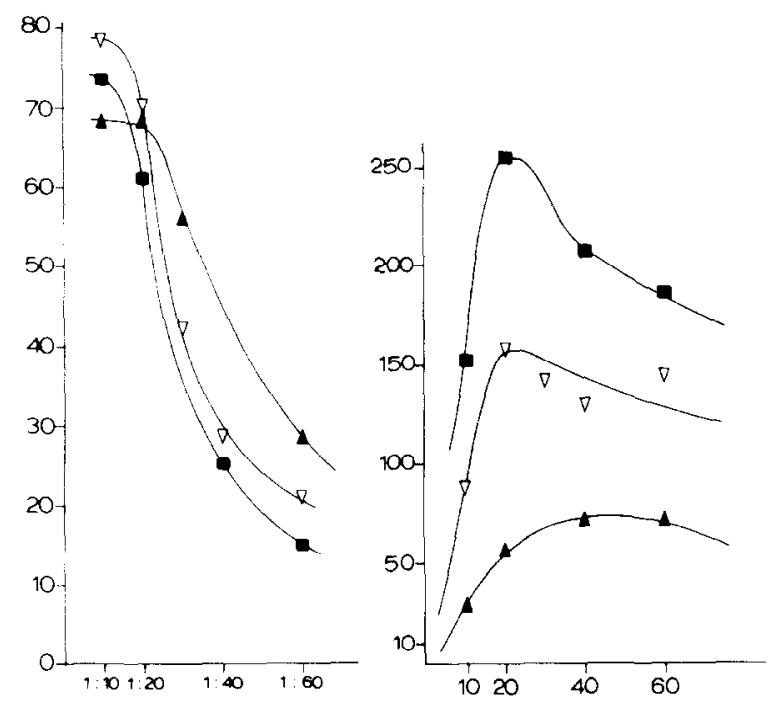

Fig. 2. Cholesterol binding by fragments of cross-linked PVAS as a function of the plasma/binder ratio $(v / v)$, using normal or type II hyperlipoproteinic defined citrated plasmas.

$A$ : Binding as percent of total amount of cholesterol brought in the incubation. $B$ : Amount of cholesterol bound to the gel fragments. Total cholesterol concentrations in these plasmas: $\Delta: 4.3 \mathrm{mM}$ (pool; $1.39 \mathrm{mM} \mathrm{TG}$ ); $\Delta: 11.2 \mathrm{mM}$ (male: $2.3 \mathrm{ml}$ TG); $: 20.7 \mathrm{mM}$ (male: $3.25 \mathrm{mM} \mathrm{TG}$ ).

plasma/binder ratio of 20/1-40/1 (by volume). At higher ratios the absolute binding decreases (Fig. 2B).

As shown in Table 1 a higher concentration of PVAS during irradiation results in a more densely cross-linked network. In order to study the effect

\section{TABLE 3}

BINDING OF APO B AND Lp(a) BY A PVAS HYDROGEL (DS $=0.73: 15 \%$ PVAS DURING $\gamma$-IRRADIATION) FROM 5 DIFFERENT SERA

Incubation of $1 \mathrm{ml}$ serum with $80 \mu 1$ PVAS gel suspension.

\begin{tabular}{lllll}
\hline $\begin{array}{l}\text { Apo B conc. \% removed a } \\
\text { before inc. } \\
(\mathrm{mg} / \mathrm{d} \mathrm{l})\end{array}$ & $\begin{array}{l}\mathrm{Lp}(\mathrm{a}) \text { conc. } \\
\text { before inc. } \\
(\mathrm{mg} / \mathrm{dl})\end{array}$ \\
\hline 160.1 & 76.2 & 83.4 & 75.1 & $(6.7)$ \\
137.2 & 70.9 & 143.8 & 40.2 & $(4.4)$ \\
145.6 & 76.4 & 93.7 & 61.1 & $(3.8)$ \\
145.6 & 73.7 & 111.0 & 74.2 & $(7.0)$ \\
131.1 & 66.7 & 89.7 & 64.9 & $(5.0)$ \\
\hline
\end{tabular}

a 1 experiment.

b 3 experiments.

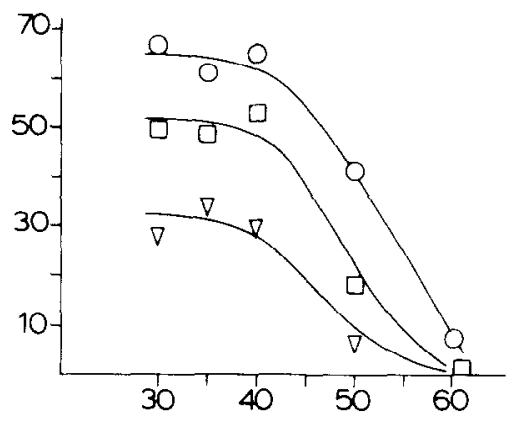

Fig. 3. Influence of concentration of PVAS (LMW, DS $=0.88$ ) during $\gamma$-irradiation, on binding of cholesterol from citrated plasma (from a local bloodbank total cholesterol $5.6 \mathrm{mM}$ ) by the resulting cross-linked, swollen and fragmentated material. Plasma/binder ratios: 20/1 (O—— O), 40/1 ( $\square \longrightarrow \longrightarrow \square)$, $60 / 1(\Delta-\Delta)$.

of the mesh size of the gel on the LDL binding we prepared a series of hydrogels with increasing concentrations PVAS during $\gamma$-irradiation (thus increasing cross-linking density). The results are summarized in Fig. 3. In this figure we can distinguish 3 different regions: (1) below 30\% PVAS no hydrogel is formed, (2) between 30 and $40 \%$ PVAS the cholesterol binding is independent of

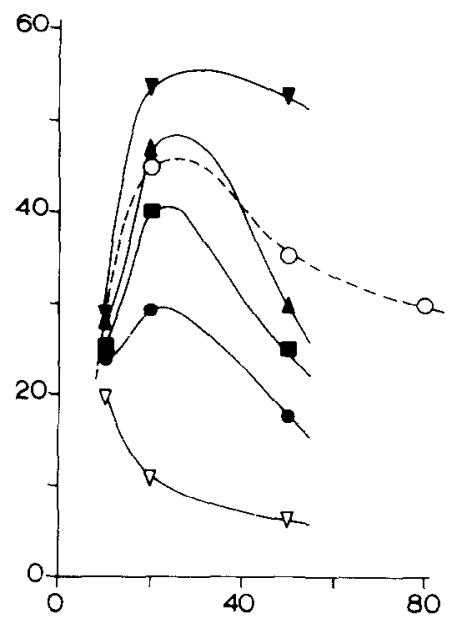

Fig. 4. Influence of binder particle size on cholesterol binding (citrated plasma from a local bloodbank); total cholesterol 4.8 $\mathrm{mM}$. Cross-linked PVAS was prepared by $\gamma$-irradiation of a $25 \%(w / w)$ solution of PVAS (LMW, DS $=0.88$ ). The resulting material was dialyzed against water, lyophilized milled and sieved as described in Material and Methods. Particle size (dry, according to the mesh of the sieves): $\nabla<45 \mu \mathrm{m}, \wedge 45-53 \mu \mathrm{m}$, - 53-63 $\mu \mathrm{m}, \quad 63-90 \mu \mathrm{m}, \nabla 90-105 \mu \mathrm{m}$, O Dounce (wet disruption). 


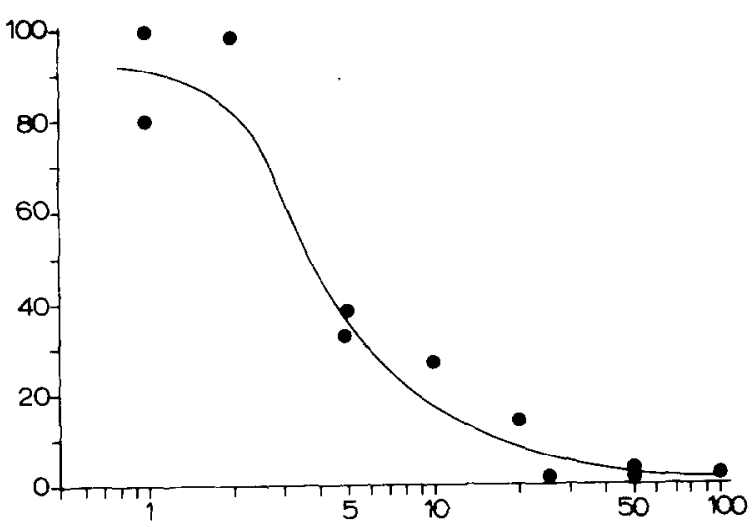

Fig. 5. Binding of antithrombin-III in citrated plasma (from the regional bloodbank in Enschede) by fragments of cross-linked PVAS. The binding is expressed as \% of the initial amount of AT-III in the plasma, and plotted as a function of the plasma/binder ratio $(\mathrm{v} / \mathrm{v})$.

the gel network mesh size, (3) at PVAS concentrations higher than $40 \%$ the mesh size of the gel becomes limiting for effective LDL penetration, thus leading to a reduced cholesterol binding.

In order to study the effect of the gel particle size on the cholesterol binding, gel particles were prepared by milling and sieving a freeze-dried hydrogel. As shown in Fig. 4 the cholesterol binding increases with decreasing gel particle size (thus increasing gel surface). With each sieve-fraction, except the coarsest, the amount of LDL cholesterol bound reached a maximum upon addition of increasing amounts of plasma and decreased again

TABLE 4

REMOVAL OF FIBRINOGEN (Fb) AND GENERATION OF FIBRINOGEN DEGRADATION PRODUCTS (FPD/ fdp) IN CITRATED PLASMA OBTAINED FROM A LOCAL BLOODBANK BY CROSS-LINKED PVAS FRAGMENTS

Mean and $\mathrm{SD}, \mathbf{n}=3$

\begin{tabular}{llll}
\hline $\begin{array}{l}\text { Plasma/ } \\
\text { binder } \\
\text { ratio } \\
(\mathrm{v} / \mathrm{v})\end{array}$ & $\begin{array}{l}\text { Fb removed } \\
\text { (mg/ml binder) }\end{array}$ & (\% of initial $\mathrm{Fb})$ & $\begin{array}{l}\text { FDP } / \mathrm{fdp} \\
\text { formed } \\
(\mu \mathrm{g} / \mathrm{ml} \\
\text { binder })\end{array}$ \\
\hline $20 / 1$ & $28.3 \pm 2.1$ & $95.9 \pm 2.5$ & $15.1 \pm 2.8$ \\
$40 / 1$ & $50.7 \pm 2.8$ & $88.0 \pm 6.4$ & $18.5 \pm 5.7$ \\
$60 / 1$ & $58.8 \pm 7.3$ & $68.1 \pm 10.3$ & $19.2 \pm 5.2$ \\
\hline
\end{tabular}

at higher amounts of plasma. The height of the maximum increased with decreasing average particle size.

The binding of other plasma components was also investigated. At a citrated plasma/binder ratio of $20 / 1,13 \%$ of the total calcium and $22 \%$ of the total magnesium was bound. At plasma/ binder ratios of $40 / 1$ and higher, divalent cation binding dropped to undetectable values. The binding of AT-III appeared to be strongly dependent on the plasma/binder ratio. Up to a plasma/binder ratio of 2 , virtually all the AT-III is bound. An increase of this ratio results in a rapid reduction of the AT-III binding, so that at plasma/binder ratios over $20 / 1$, less than $10 \%$ of the AT-III is bound by the PVAS hydrogel (Fig. 5). Albumin and $\alpha_{1^{-}}, \alpha_{2^{-}}, \beta$ - and $\gamma$-globulins were not appreciably bound to the gel fragments in incubations with plasma/binder ratios of $20 / 1$ or higher (not shown). Fibrinogen, however, was largely removed from citrated plasma in such incubations, which could be ascribed to extensive $\mathrm{Fb}$ degradation (Table 4).

\section{Discussion}

Loosely cross-linked, highly sulfated PVA hydrogels are able to bind LDL selectively and with high capacity from blood plasma. The LDL binding is reduced by very high VLDL levels (type IV HLP) and at very high plasma/binder ratios by other plasma factors*.

The LDL binding also depends on the mesh size of the network (a too finely meshed network inhibits LDL penetration into the gelmatrix) and on the particle size of the binder i.e. the total binder surface. The effects of both gel surface and degree of cross-linking on LDL binding indicate a restricted penetration of the LDL particle into the gelmatrix. Theoretical calculations for LDL penetration into the gelmatrix predict a penetration depth of LDL in the matrix of $10 \mu \mathrm{m}$. This means that only part of the total gel volume of the particles used is actually involved in the LDL

\footnotetext{
* In separate experiments binding of isolated LDL was found to be inhibited by addition of the dialyzed $d>1.21 \mathrm{~g} / \mathrm{ml}$ ultracentrifugation fraction of serum or citrated plasma (H.J.M. Kempen, unpublished observation).
} 
binding process. Therefore the LDL binding capacity could theoretically be increased by further reducing the gel particle size, but this will probably introduce problems in filtering the gel from the treated plasma.

In addition to LDL, these gels bind a cholesterol rich subfraction from the $d<1.019 \mathrm{~g} / \mathrm{ml}$ lipoproteins. A similar cholesterol-rich VLDL subfraction was isolated from the total $d<1.006 \mathrm{~g} / \mathrm{m}$ lipoproteins by passage through a column of heparin-sepharose [14]. Removal of these particles can be considered as favourable, since these particles are thought to be atherogenic [15]. Furthermore $\mathrm{Lp}(\mathrm{a})$, also considered as an atherogenic particle [16], is bound to a considerable extent, whereas $\mathrm{HDL}_{2}$ and $\mathrm{HDL}_{3}$ are virtually not adsorbed. Binding of other plasma proteins was also investigated. Binding of AT-III, albumin and various globulins was less than $10 \%$ at plasma/binder ratios above $20 / 1$. Fibrinogen was largely removed, however mostly by degradation. This loss of fibrinogen is caused by extensive activation of Factor XII and prekallikrein by the cross-linked PVAS fragments (G. Dooijewaard and C. Kluft, unpublished), thus initiating both clotting and fibrinolysis cascades in the citrated plasma.

Compared to other LDL binders, such as immobilized heparin or dextran sulfate, this binder has a very high capacity for LDL binding. As shown in Fig. 2 the binder removes almost all the LDL from 10 to 20 times its own volume of plasma, even when plasma with extremely elevated LDL levels is used. Therefore, LDL plasma levels in FH patients could theoretically be lowered by $90 \%$ with only $150-300 \mathrm{ml}$ binder particles. In a clinical situation, however, the LDL reduction will be less pronounced due to the limitations in extracorporeal blood volume (about $500 \mathrm{ml}$ ) and also due to the dilution of untreated with treated blood, resulting in a logarithmic decrease of the LDL level.

In contrast to immobilized heparin and dextran sulfate, no additives such as divalent cations are required for LDL removal by cross-linked PVAS particles, which not only simplifies clinical procedures, but also permits the use of citrate-based anticoagulants.

The results of these experiments suggest that an extracorporeal plasma treatment with sulfated polyvinylalcohol gels could offer a new and useful technique for lowering LDL levels of FH patients. Further research will be focussed on the biocompatibility of this new LDL binding material, as well as on the manner in which it can be incorporated in a device for extracorporeal LDL apheresis.

\section{Acknowledgements}

We thank Mrs. R. van Schie-Erades, Mrs. B. Hoegee and Mr. H. Van der Voort for excellent technical assistance; Dr. W. Nieuwenhuizen for determination of fibrinogen and fibrinogen degradation products; Dr. R. Zechner, Department of Biochemistry, University of Graz, Austria, for determination of the apo $B$ and $L p(a)$ concentrations; Mrs. L. Rotman for typing the manuscript and Drs. J.A. Gevers Leuven and G. Thompson for supply of plasma from different HLP patients.

\section{References}

1 Thompson, G.R., Plasma lipoproteins and their disorders, Medicine (Baltimore), 11 (1978) 558

2 AHA Special Report, Recommendations for treatment of hyperlipidemia in adults, Circulation, 69 (5) (1984) 1065A.

3 Thompson, G.R., Plasma exchange and affinity chromatographic therapy for hyperlipidaemia - A review, Apheresis Bulletin, 1 (1983) 26.

4 Lupien, P.J., Moorjani. S., Gagne, C., Brun, B.R., Lou, M. and Dagenais, G., Long term treatment of two FH.He patients with batch affinity chromatography. Artery 10 (4) (1982) 286.

5 Stoffel. W., Greve, V. and Borberg. H., Application of specific extracorporeal removal of LDL in FH. Lancet, Nov. 7 (1981) 1005.

6 Fuchs, Ch., Windisch, M., Wieland, H., Armstrong, V.W.. Riegel, J., Köstering, H., Scheler, F. and Seidel, D., Selective continuous extracorporeal elimination of low density lipoproteins from plasma by heparin precipitation without cations. In: M.J. Lysaght and H.J. Gurland (Eds.), Plasma Separation and Plasma Fractionation, Karger, Basel, 1983. pp. 272.

7 Redgrave, T.G., Roberts, D.C.K. and West, C.E., Separation of plasma lipoproteins by density gradient ultracentrifugation, Anal. Biochem., 65 (1975) 42.

8 Abell, L.L., Levy, B.B., Brodie, B.B. and Kendall, F.E., A simplified method for the estimation of total cholesterol in scrum and demonstration of its specificity. J. Biol. Chem., 195 (1952) 357

9 Giegel, J.L., Ham, A.B. and Clema, W., Manual and semi-automated procedures for measurement of triglycerides in serum, Clin. Chem., 21 (1975) 1575. 
10 Laurell, C.B., Quantitative estimation of proteins by electrophoresis in agarose gel containing antibodies, Anal. Biochem., 15 (1966) 45.

11 Mancini, G., Carbonara, A.O, and Heremans, J.F.., Immunochemical quantitation of antigens by simple radial immunodiffusion, Immunochemistry, 2 (1965) 235.

12 Koppert, P.W., Huijsmans, C.M.G. and Nieuwenhuizen, W., A monoclonal antibody, specific for human fibrinogen, fibrinopeptide A-containing fragments, and not reacting with free fibrinopeptide A, Blood, 66 (1985) 503.

13 Nieuwenhuizen, W., Koppert, P.W., Haverkate, F. and Koopmans, J., A monoclonal antibody specific for fibrin (ogen) degradation products (FDP/fdp). Residues B-beta 54-118 present a FDP/fdp-specific neoantigenic determinant, Thromb. Haemost., 54 (1985) 47.

14 Huff, M.W. and Telford, D.E., Characterization and metabolic fate of two very-low-density lipoprotein subfractions separated by heparin-sepharose chromatography, Biochim. Biophys. Acta, 796 (1984) 251.

15 Zilversmit, D.B., Atherogenesis: a postprandial phenomenon, Circulation, 60 (1979) 473

16 Berg, K., Dahlen, G. and Frick, M.H., Lp(a) lipoprotein and pre- $\beta_{1}$-lipoprotein in patients with coronary heart diseases, Clin. Genet., 6 (1974) 230. 